\title{
Influence of interface termination on the magneto-Seebeck effect in MgO based tunnel junctions
}

\author{
Michael Czerner and Christian Heiliger ${ }^{a)}$ \\ I. Physikalisches Institut, Justus Liebig University, Giessen, Germany
}

(Presented 2 November 2011; received 22 September 2011; accepted 7 November 2011; published online 1 March 2012)

On an ab initio level, we investigate the recently observed magneto-Seebeck effect in $\mathrm{MgO}$ based tunnel junctions. In particular, we considered ordered CoFe alloys as lead material. Next to the $\mathrm{MgO}$ barrier, there are different possible terminations of the CoFe alloy due to the assumed ordered alloy. These results show a strong influence of the termination on the temperature dependence of the magneto-Seebeck effect. In addition, we use a simple model to account for randomly ordered alloys. We propose, that by a controlled treatment of the $\mathrm{CoFe} / \mathrm{MgO}$ interface the magneto-Seebeck effect can be tuned experimentally. (C) 2012 American Institute of Physics. [doi:10.1063/1.3675987]

\section{INTRODUCTION}

The magneto-Seebeck effect is one effect in the new field of spincaloritronics, ${ }^{1}$ which is the combination of two research fields: magnetism (spintronics) and thermoelectric. The effect describes the dependence of the Seebeck coefficient (or thermopower) in a spin valve or tunnel junction on the relative magnetic orientation of the ferromagnetic leads. This way the effect is similar to giant magneto resistance (GMR) and tunnel magneto resistance (TMR) but for the thermopower instead of electrical resistance.

The effect was experimentally observed in GMR structures $^{2}$ and was theoretically predicted to be high in $\mathrm{MgO}$ based tunnel junctions. ${ }^{3}$ Recent experiments measured the magneto-Seebeck effect in $\mathrm{MgO}$ based tunnel junctions with a $\mathrm{CoFe}$ alloy as ferromagnets. ${ }^{4,5}$ However, the experiments as well as the presented theoretical results for different interface terminations of the CoFe alloy were discussed at room temperature only. In this paper, we discuss the influence of the termination of the CoFe alloy on the temperature dependence of the magneto-Seebeck effect. In addition, we propose in the last section a simple model to account for disordered alloys. The different structures of the ordered alloy discussed in the following are sketched in Fig. 1. We assume ordered alloys with alternating monolayers of $\mathrm{Fe}$ and Co. Therefore, only the three different junctions shown in Fig. 1 are possible.

\section{METHOD}

We use a Korringa-Kohn-Rostoker (KKR) method based on density functional theory to calculate the electronic structure of the junctions shown in Fig. 1 self-consistently. For the transport calculation, we evaluate the transmission function $T(E)$ first. $T(E)$ is used to calculate the moments

$$
L_{n}=\frac{2}{h} \int T(E)(E-\mu)^{n}\left(-\partial_{E} f(E, \mu, T)\right) d E,
$$

\footnotetext{
${ }^{a)}$ Electronic mail: christian.heiliger@physik.uni-giessen.de.
}

where $f(E, \mu, T)$ is the Fermi function at a given energy $E$, electrochemical potential $\mu$, and temperature $T$. Eventually, the conductance $G$ and the Seebeck coefficient $S$ are given by ${ }^{9}$

$$
G=e^{2} L_{0} S=-\frac{1}{e T} \frac{L_{1}}{L_{0}} .
$$

The transmission function $T(E)$ is calculated using the nonequilibrium Green's function (NEGF) formalism implemented in KKR. ${ }^{6}$ The temperature dependence is considered within the Fermi function only. Although this treatment is a crucial simplification, it is justified by the experimental observation that there is only a small influence of the temperature on the TMR effect. ${ }^{7}$ Further, theoretical investigations of tunnel junctions with amorphous iron leads show that the transport properties are determined mainly by the crystalline iron layers next to the $\mathrm{MgO}$ barrier. $^{8}$ In particular, the size and orientation of the magnetization as well as inelastic effects farther away from the $\mathrm{MgO}$ barrier are of second order influence. All the numerical parameters are the same like in our previous study. ${ }^{3}$ The calculation of the Seebeck coefficient is done for parallel (P) and anti-parallel (AP) alignment of the ferromagnetic leads to calculate the magneto-Seebeck $S_{M S}$ effect by

$$
S_{M S}=\frac{S_{P}-S_{A P}}{\min \left(\left|S_{P}\right|,\left|S_{A P}\right|\right)} .
$$

\section{RESULTS}

\section{A. Ordered alloy}

In Fig. 2, we show the calculated magneto-Seebeck and the Seebeck coefficients for parallel and anti-parallel alignment for the junctions shown in Fig. 1 for two different barrier thicknesses. The viewgraphs clearly show that there is a strong influence of the termination of $\mathrm{CoFe}$ on the magneto-Seebeck effect. A similarity is that all $S_{M S}$ are 

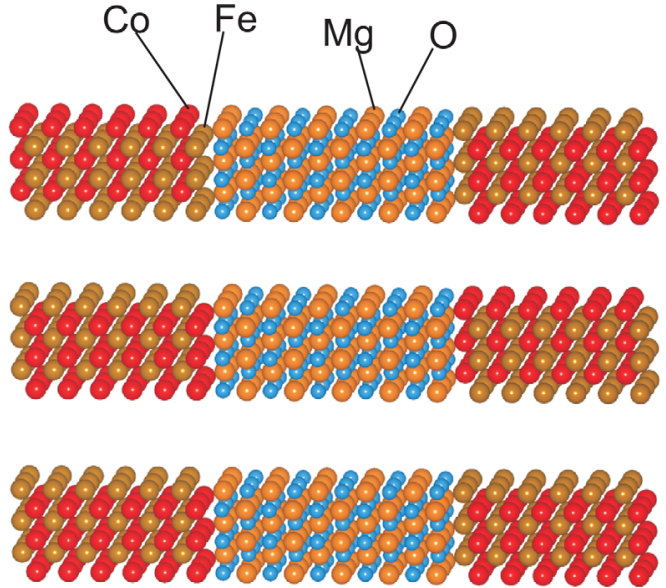

FIG. 1. (Color online) Different possible structures assuming ferromagnetic leads to alternating monolayer of $\mathrm{Fe}$ and $\mathrm{Co}$. The thickness of the $\mathrm{MgO}$ barrier is 6 or 10 monolayers and of the CoFe layer is 20 monolayers. These structures are embedded between semi-infinite $\mathrm{Cu}$ in $\mathrm{Fe}$-bcc structure, which acts as reservoirs.

positive at all temperatures, but the magnitude is quite different. In particular, the junction with different termination left and right has the highest values whereas the Co terminated junction has the smallest values.

Another common feature is the minimum of the graphs, which is around room temperature. Therefore, in experiments one has to go to larger or lower temperatures to increase $S_{M S}$. However, the calculations are not considering any temperature effects besides occupation. Therefore, the calculated values at higher temperatures have to be seen with caution. By making the barrier thicker, no general trend is observed. For mixed termination and for Fe termination, the
magneto-Seebeck is decreasing by increasing the barrier thickness whereas for the Co terminated junction it is the opposite behavior.

The magneto-Seebeck with $\mathrm{Fe}$ termination show a divergence at a temperature of about $150 \mathrm{~K}$. Such a divergence can be addressed either to a vanishing Seebeck coefficient parallel or anti-parallel. Figure 2 shows that the Seebeck coefficients for the anti-parallel alignment are always negative and have an overall trend with a maximum at about room temperature. This maximum corresponds to the observed minimum in the magneto-Seebeck. Consequently, the origin of the divergence lays in a vanishing Seebeck coefficient in parallel alignment. Interestingly, at a larger barrier thickness, the divergence vanishes.

\section{B. Disordered alloy}

Up to now, we considered only ordered alloys. Experimentally, disordered alloys are more realistic. However, the discussed dependencies of the last section could be obtained by controlling the termination of the interface layer. In order to estimate the magneto-Seebeck effect in disordered alloys, we apply the simple model proposed in Ref. 10 . The idea is that in a disordered alloy there are still the junctions of Fig. 1 present and we can assume that the transport through the whole junction is simply given by conducting the microscopic junctions parallel.

In particular, if we have a disordered alloy of $50 \%$ Co and $50 \% \mathrm{Fe}$, the probability of having junction (1) or (2) is $25 \%$ each and of having junction (3) (including the inverted one) is $50 \%$. Conducting in parallel means that the transmission functions are just added up. Consequently, the total transmission function of a disordered alloy is
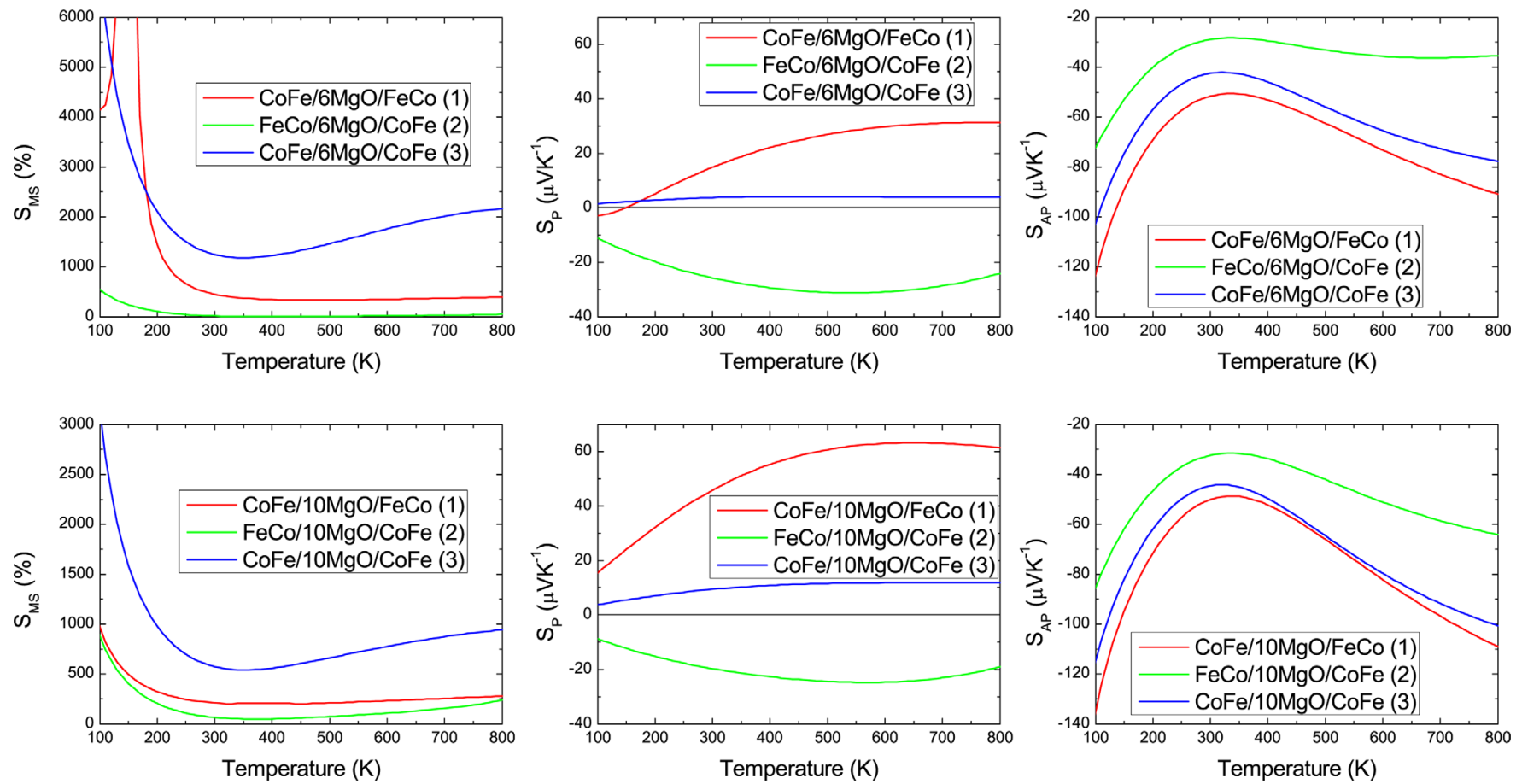

FIG. 2. (Color online) Magneto-Seebeck $S_{M S}$ (left column) and Seebeck coefficients for parallel (middle column) and anti-parallel (right column) alignment. Top: a barrier thickness of 6 monolayers MgO. Bottom: a barrier thickness of 10 monolayers MgO. The numbering of the graphs corresponds to the junctions shown in Fig. 1. 


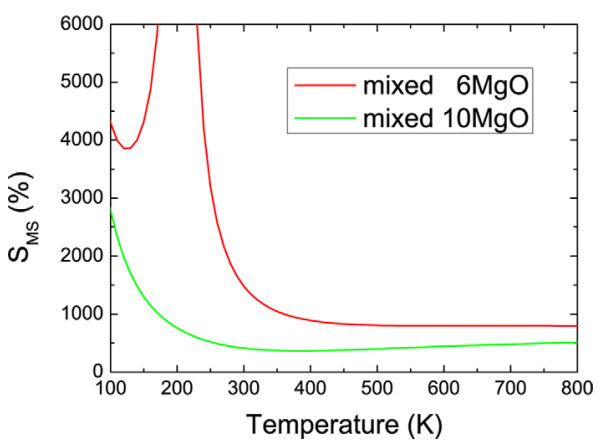

FIG. 3. (Color online) Magneto-Seebeck for disordered alloy $S_{\text {dis }}$ (Eq. (5)) as a function of temperature. Assumed is an equal random distribution of $\mathrm{Fe}$ and $\mathrm{Co}$ at the interface, e.g., $P_{1}=P_{2}=25 \%, P_{3}=50 \%$ in Eq. (5).

$$
T_{d i s}(E)=P_{1} T_{1}(E)+P_{2} T_{2}(E)+P_{3} T_{3}(E),
$$

where $P_{i}$ is the probability to find junction $\mathrm{i}$ in the disordered alloy. Using this transmission function in Eq. (2) leads to the Seebeck coefficient in the disordered system

$$
S_{d i s}=\frac{P_{1} S_{1} G_{1}+P_{2} S_{2} G_{2}+P_{3} S_{3} G_{3}}{P_{1} G_{1}+P_{2} G_{2}+P_{3} G_{3}} .
$$

Figure 3 shows the magneto-Seebeck for the case $P_{1}=P_{2}=25 \%, P_{3}=50 \%$. The important feature of this viewgraph is that the magneto-Seebeck is also always positive for all temperatures like in the case of the ordered alloys. On one hand, experiments by Liebing et al. ${ }^{5}$ show positive magneto-Seebeck effect. On the other hand, other measurements and previous calculations with an ordered CoFe inplane supercell show negative magneto-Seebeck effect. ${ }^{4}$ Obviously, our proposed model Eq. (5) is too simplistic to account for a disordered alloy. Improvements could be the use of different in-plane supercells and averaging them or the use of advanced methods of alloy theory. The latter can be achieved within KKR by using, e.g., coherent potential approximation (CPA).

\section{CONCLUSION}

The termination of the CoFe alloy next to the $\mathrm{MgO}$ barrier influences the magneto-Seebeck effect drastically. For $\mathrm{Fe}$ termination at both sides of $\mathrm{MgO}$ even a divergence occurs for 6 monolayers of $\mathrm{MgO}$. Interestingly, this divergence vanishes going to thicker $\mathrm{MgO}$ barrier. The proposed simple model to extrapolate from the ordered alloy to a disordered alloy was not able to capture the experimentally observed negative magneto-Seebeck effect. In the future, we plan to improve our method by using alloy theory (KKRCPA) to account for disordered alloys.

\section{ACKNOWLEDGMENTS}

We acknowledge support from German Research Foundation via SPP 1538 "SpinCaT."

${ }^{1}$ G. E. W. Bauer, A. H. MacDonald, and S. Maekawac, Solid State Commun. 150, 459 (2010).

${ }^{2}$ L. Gravier, S. Serrano-Guisan, F. Reuse, and J. P. Ansermet, Phys. Rev. B 73, 024419 (2006).

${ }^{3}$ M. Czerner, M. Bachmann, and C. Heiliger, Phys. Rev. B 83, 132405 (2011).

${ }^{4}$ M. Walter, J. Walowski, V. Zbarsky, M. Münzenberg, M. Schäfers, D. Ebke, G. Reiss, A. Thomas, P. Peretzki, M. Seibt, J. S. Moodera, M. Czerner, M. Bachmann, and C. Heiliger, Nature Mater. 10, 742 (2011).

${ }^{5}$ N. Liebing, S. Serrano-Guisan, K. Rott, G. Reiss, J. Langer, B. Ocker, and H. W. Schumacher, Phys. Rev. Lett. 107, 177201 (2011)

${ }^{6}$ C. Heiliger, M. Czerner, B. Yu. Yavorsky, I. Mertig, and M. D. Stiles, J. Appl. Phys. 103, 07A709 (2008).

${ }^{7}$ S. Yuasa, T. Nagahama, A. Fukushima, Y. Suzuki, and K. Ando, Nature Mater. 3, 868 (2004).

${ }^{8}$ M. Gradhand, C. Heiliger, P. Zahn, and I. Mertig, Phys. Rev. B 77, 134403 (2008).

${ }^{9}$ Y. Ouyang and J. Guo, Appl. Phys. Lett. 94, 263107 (2009).

${ }^{10}$ C. Heiliger, P. Zahn, and I. Mertig, J. Magn. Magn. Mater. 316, 478 (2007). 